Accepted by AJ on 2016 April 12

\title{
A Very Large Array Search for Intermediate-Mass Black Holes in Globular Clusters in M81
}

\author{
J. M. Wrobel ${ }^{1,2}$, J. C. A. Miller-Jones ${ }^{3}$, and M. J. Middleton ${ }^{4}$
}

\begin{abstract}
Nantais et al. used the Hubble Space Telescope to localize probable globular clusters (GCs) in M81, a spiral galaxy at a distance of $3.63 \mathrm{Mpc}$. Theory predicts that GCs can host intermediate-mass black holes (IMBHs) with masses $M_{\mathrm{BH}} \sim$ 100-100, $000 M_{\odot}$. Finding IMBHs in GCs could validate a formation channel for seed BHs in the early universe, bolster gravitational-wave predictions for space missions, and test scaling relations between stellar systems and the central BHs they host. We used the NRAO Karl G. Jansky Very Large Array (VLA) to search for the radiative signatures of IMBH accretion from 206 probable GCs in a mosaic of M81. The observing wavelength was $5.5 \mathrm{~cm}$ and the spatial resolution was $1.5^{\prime \prime}$ (26.4 pc). None of the individual GCs are detected, nor are weightedmean image stacks of the 206 GCs and the 49 massive GCs with stellar masses $M_{\star} \gtrsim 200,000 M_{\odot}$. We apply a semi-empirical model to predict the mass of an IMBH that, if undergoing accretion in the long-lived hard X-ray state, is consistent with a given radio luminosity. The $3 \sigma$ radio-luminosity upper limits correspond to IMBH masses of $\overline{M_{\mathrm{BH}} \text { (all) }}<42,000 M_{\odot}$ for the all-cluster stack and $\overline{M_{\mathrm{BH}}(\text { massive })}<51,000 M_{\odot}$ for the massive-cluster stack. We also apply the empirical fundamental-plane relation to two X-ray-detected clusters, finding that their individual IMBH masses at $95 \%$ confidence are $M_{\mathrm{BH}}<99,000 M_{\odot}$ and $M_{\mathrm{BH}}<15,000 M_{\odot}$. Finally, no analog of HLX-1, a strong IMBH candidate in an extragalactic star cluster, occurs in any individual GC in M81. This underscores the uniqueness or rarity of the HLX-1 phenomenon.
\end{abstract}

\footnotetext{
${ }^{1}$ National Radio Astronomy Observatory, P.O. Box O, Socorro, NM 87801, USA; jwrobel@nrao.edu

${ }^{2}$ National Science Foundation, 4201 Wilson Boulevard, Arlington, VA 22230, USA; jwrobel@nsf.gov

${ }^{3}$ International Centre for Radio Astronomy Research, Curtin University, GPO Box U1987, Perth, WA 6845, Australia; james.miller-jones@curtin.edu.au

${ }^{4}$ Institute of Astronomy, University of Cambridge, Madingley Road, Cambridge CB3 0HA, UK; mjm@ast.cam.ac.uk
} 
Subject headings: black hole physics — galaxies: individual (M81) — galaxies: star clusters: individual (M81, ESO 243-49 HLX-1, M60-UCD1) — radio continuum: general

\section{Motivation}

Intermediate-mass black holes (IMBHs) are thought to occupy the mass gap between the well-established stellar-mass BHs with $M_{\mathrm{BH}}<100 M_{\odot}$ (e.g., Abbott et al. 2016a,b; Corral-Santana et al. 2016; Tetarenko et al. 2016) and the well-studied supermassive BHs with $M_{\mathrm{BH}} \gtrsim 10^{6} M_{\odot}$ (e.g., Kormendy \& Ho 2013). Finding IMBHs in the local universe can provide important insight into formation channels for seed BHs in the early universe (for reviews, see Greene 2012; Volonteri 2012; Natarajan 2014). Here, we focus on theoretical predictions that globular clusters (GCs) could host IMBHs formed via dynamical processes stemming from the clusters' closely packed stars (e.g., Miller \& Hamilton 2002; Gurkan et al. 2004; Portegies Zwart et al. 2004; Giersz et al. 2015). In addition, if IMBHs exist in GCs, inspirals involving them and stellar-mass BHs could be years-long sources of gravitational waves for a LISA-like mission (Konstantinidis et al. 2013). Such IMBHs could also be used to test scaling relations between central BHs and their stellar system hosts, thus informing the debate about whether or not these entities co-evolve (e.g., Xiao et al. 2011; Jiang et al. 2011; Kormendy \& Ho 2013; Graham et al. 2016).

A key science driver for future near-infrared, ground-based telescopes is to measure, at a distance of $10 \mathrm{Mpc}$, a $\mathrm{BH}$ mass as low as $M_{\mathrm{BH}} \sim 10^{5} M_{\odot}$ by spatially resolving its sphere of influence in its host stellar system (Do et al. 2014). It is expected that this will yield a robust inventory of IMBHs in GCs in the local universe, but these next-generation facilities are many years off. Moreover, although GCs in the Local Group have been targeted in sphereof-influence studies, all these studies are contentious (Strader et al. 2012a, and references therein).

Given these controversies and long waits for future facilities, we have begun an independent investigation, namely searching for radio signatures of accretion onto putative IMBHs in extragalactic GCs (Wrobel et al. 2015). By analogy with stellar-mass BHs (reviewed by Fender \& Belloni 2012), one expects that an IMBH will spend more time in the hard X-ray state - including quiesence - associated with a low accretion rate onto the $\mathrm{BH}$, than in the soft X-ray state associated with a high accretion rate. In the typical case of only a few radio observations, it is likely that they will sample the steady radio emission characteristic of the hard X-ray state, as opposed to the flaring radio emssion associated with a transition from the hard X-ray state to the soft X-ray state. These concepts, first laid out by Maccarone 
(2004), lead to the following three approaches:

(1) Detect radio emission like that from HLX-1 in its hard X-ray state, where HLX-1 is a strong IMBH candidate in an extragalactic star cluster with a stellar mass of $M_{\star} \sim$ $10^{5-6} M_{\odot}($ Cseh et al. 2015).

(2) Use the empirical fundamental-plane regression for the hard X-ray state, plus observations of X-ray and radio luminosities, to estimate an IMBH mass (Merloni et al. 2003; Falcke et al. 2004; Plotkin et al. 2012).

(3) Use a conservative, semi-empirical model to predict the mass of an IMBH that, if experiencing Bondi accretion in the hard X-ray state, would be consistent with the observed radio luminosity (Maccarone \& Servillat 2008, 2010; Strader et al. 2012a).

Our radio imaging of NGC1023, an early-type galaxy about $11 \mathrm{Mpc}$ away, detected none of its 337 candidate star clusters (Wrobel et al. 2015). The approaches above then led to the following inferences: (1) No HLX-1 analogs suggests that phenomenon is very rare or is fuelled from gas related to its cluster's relatively young stars. (2) To be able to reach the regime of IMBH masses, deeper X-ray and radio surveys that detect candidate clusters at lower luminosities are needed. Importantly, the mass term in the fundamental plane implies that, in the hard-X-ray state, the radio emission from IMBHs should be much brighter than that from stellar-mass BHs. This makes radio detections effective at filtering out contamination from the X-ray-emitting stellar-mass BHs often found in star clusters. (3) The radio-luminosity upper limit for a stack of the 20 most massive clusters corresponds to a mean $3 \sigma \mathrm{IMBH}$ mass of $\overline{M_{\mathrm{BH}} \text { (massive) }}<2.3 \times 10^{5} M_{\odot}$ and a $\mathrm{BH}$ mass fraction $\overline{M_{\mathrm{BH}}(\text { massive })} / \overline{M_{\star}(\text { massive })}<0.16$.

The inferences from our NGC 1023 study can be improved upon by obtaining longer exposures on star clusters in closer galaxies. We thus turn to M81, a spiral galaxy at a distance of $3.63 \pm 0.34 \mathrm{Mpc}\left(1^{\prime \prime}=17.6 \mathrm{pc}\right.$; Freedman et al. 1994) that is estimated to have 210 \pm 30 GCs in total (Perelmuter \& Racine 1995). Several studies have used the Hubble Space Telescope (HST) to localize candidate GCs in M81 (Chandar et al. 2001, 2004; Nantais et al. 2010; Santiago-Cortes et al. 2010; Nantais et al. 2011). Here, we focus on the Nantais et al. (2011) study because it leveraged Advanced Camera for Surveys (ACS) mosaics with spectroscopy to identify probable GCs in M81. Nantais et al. (2011) performed aperture photometry and profile fitting on 419 candidate GCs, and highlight $214^{1}$ as being

\footnotetext{
${ }^{1}$ Nantais et al. (2011) tabulate this number but mistakenly quote 221 in their text.
} 
probable GCs because they were spectroscopically confirmed (85) or were good candidates (129) that shared the color and size ranges of the spectroscopically confirmed GCs. The V-band (F606W) magnitudes from Nantais et al. (2011) and that band's mass-to-light ratio for GCs from Harris et al. (2010) imply that the stellar masses of the 214 probable GCs range from $M_{\star} \sim 1 \times 10^{4} M_{\odot}$ to $M_{\star} \sim 8 \times 10^{6} M_{\odot}$.

In this paper, we use the $\mathrm{NRAO}^{2}$ Karl G. Jansky Very Large Array (VLA; Perley et al. 2011) to search for radio emission from these probable GCs in M81. We describe our new VLA results in $\S 2$. No individual probable GC is detected. The implications of these nondetections are then explored regarding HLX-1 analogs (§ 3.1), X-ray detected clusters (§ 3.2), and semi-empirical model predictions ( $\S 3.3)$. We close in $\S 4$ with a summary and conclusions.

\section{Imaging}

We observed M81 under proposal code 13B-138 (PI M. Middleton) using the VLA in its B configuration at a central frequency of $5.5 \mathrm{GHz}$, corresponding to a wavelength of $5.5 \mathrm{~cm}$. Owing to the large angular size of the galaxy, we used four pointing centers separated by 4.5 to provide relatively uniform sensitivity to the central regions of the galaxy. The pointing centers' locations and observation dates appear in Table 1.

Each VLA pointing was observed for one hour, giving $41 \mathrm{~m}$ of time on target, using a correlator integration time of $3 \mathrm{~s}$. The target elevation lay between $23^{\circ}$ and $32^{\circ}$ in all cases. The observing bandwidth was split into two $1024-\mathrm{MHz}$ basebands centred at 5.0 and $6.0 \mathrm{GHz}$, each comprised of eight spectral windows of width $128 \mathrm{MHz}$, each of which was split into sixty-four 2-MHz channels. We used 3C 147 to set the amplitude scale to an estimated accuracy of about $3 \%$, and J1048+7143 as the secondary calibrator to derive the atmospheric and instrumental complex gains on a per-antenna basis. The position assumed for J1048+7143 was $\alpha(J 2000)=10^{h} 48^{m} 27^{\mathrm{s}} .6199$ and $\delta(J 2000)=71^{\circ} 43^{\prime} 35^{\prime \prime} .938$ with onedimensional errors at $1 \sigma$ of 2 mas. Given the observing strategies, the one-dimensional astrometric error at $1 \sigma$ is estimated to be 0 "' 1 .

We processed the data using standard procedures within the Common Astronomy Software Application (CASA; McMullin et al. 2007) release 4.1.0. Following external gain calibration, the Stokes $I$ data for each pointing were imaged with the CASA task clean using a

\footnotetext{
${ }^{2}$ The National Radio Astronomy Observatory is a facility of the National Science Foundation, operated under cooperative agreement by Associated Universities, Inc.
} 
robustness parameter of 0.5 to obtain the best compromise among sensitivity, spatial resolution and sidelobe suppression; an nterms parameter of 2 to accommodate the large fractional bandwidth; and the gridmode parameter set to "widefield" and the wprojplanes parameter set to 128 to correct for the effects of non-coplanar baselines. The images were dominated by the emission from the low-luminosity active galactic nucleus (LLAGN) in M81, and required self-calibration, initially in phase, and then in amplitude and phase, down to a timescale as short as the correlator integration time of $3 \mathrm{~s}$. The final, self-calibrated images of all four quadrants were restored with the same circular Gaussian restoring beam of FWHM of 1.5, and then mosaicked together, cutting off the response of the primary beam at the $10 \%$ level. In the mosaic, the LLAGN is point-like and has a flux density of $81 \pm 3$ mJy. Figure 1 shows the geometry of the VLA mosaic overlaid on a $K_{s}$ image of M81 (Jarrett et al. 2003) retrieved from NED.

For each of the 419 candidate GCs, task subim in the 2016 December 31 release of NRAO's Astronomical Image Processing System (AIPS; Greisen 2003) was used to form a cutout spanning 30" and centered on the optical position (Nantais et al. 2011). Astrometric comparions between the ACS images and ground-based surveys implied conservative onedimensional errors at $1 \sigma$ of $0^{\prime \prime} 2$ (Nantais et al. 2011). Thirteen candidate GCs lay beyond the boundary of the VLA mosaic. This left 406 candidates for analysis, split as 206 probable GCs and 200 improbable GCs. The term "improbable" refers to candidate GCs that Nantais et al. (2011) judged to be background galaxies or non-GC objects like young clusters and HII regions, or confirmed as galaxies or non-GC objects via spectroscopy.

Figure 2 shows the VLA cutouts for the 206 probable GCs. The $1 \sigma$ rms noise level among the cutouts varies from 4.3 to $51 \mu \mathrm{Jy} \mathrm{beam}^{-1}$, a range expected given the primary beam cutoff invoked. An individual one-tail detection threshold of $3 \sigma$ is adopted to minimize the risk of a false-positive detection of one or more targets when examining the ensembles of 206 probable GCs and 200 improbable GCs (Wall \& Jenkins 2003).

Only one of the 200 improbable GCs, ID 146 from Nantais et al. (2011), was detected with enough significance to serve as a crosscheck of the radio and optical astrometry. The VLA cutout of ID 146 appears in Figure 3. The radio emission is somewhat resolved but dominantly compact. The radio peak has a total offset of $0{ }^{\prime \prime} 2$ when compared to the optical position reported by Nantais et al. (2011). Assuming that the peaks should physcially coincide, such an offset is consistent with the error estimates cited above for the radio and optical data. Moreover, finding resolved radio emission from ID 146 is consistent with its classification as an improbable GC: if it was a probable GC hosting an IMBH in the low-hard state, any radio emission should be spatially unresolved. 


\section{Implications}

From Figure 2, none of the 206 probable GCs in M81 is detected above its local $3 \sigma$ level at $5.5 \mathrm{GHz}$. The associated radio luminosities are $\nu L_{\nu}=L_{\mathrm{R}}<1.1-13 \times 10^{33} \mathrm{erg}$ $\mathrm{s}^{-1}$, using a definition that implicitly assumes a flat radio continuum spectrum up to 5.5 GHz. Figure 4 conveys the radio luminosities and stellar masses of each of the 206 probable GCs. At these radio luminosities, accreting stellar-mass compact objects in the hard X-ray state would have radio luminosities too faint to be detected (Strader et al. 2012b). What about contamination from flaring radio emssion associated with a transition from the hard $\mathrm{X}$-ray state to the soft X-ray state? Indeed, such flaring is thought to be the explanation for the stellar-mass BH in M31 that achieved a peak 5-GHz luminosity of $L_{\mathrm{R}} \sim 5.3 \times 10^{33}$ $\mathrm{erg} \mathrm{s}^{-1}$ and then decayed on a timescale of days (Middleton et al. 2013, 2014). However, the lack of radio detections in Figure 4 implies that such flaring emission from stellar-mass BHs cannot be a major contaminant for these single-epoch observations of the probable GCs in M81. (Stronger inferences about flares will be reported elsewhere in conjuction with radio monitoring observations.) This paves the way for us to interpret Figure 4 within the context of the three approaches mentioned in $\S 1$, namely analogs of HLX-1 (§ 3.1), X-ray detected clusters ( $(3.2)$, and predictions of a semi-empirical model ( $(3.3)$. In the analysis to follow, it is important to keep in mind that only $40 \%$ of the probable GCs have been spectroscopically confirmed (Nantais et al. 2011).

\subsection{HLX-1 Analogs}

HLX-1 is a strong IMBH candidate, of mass $M_{\mathrm{BH}} \sim 10^{4-5} M_{\odot}$, in an extragalactic star cluster with an observed stellar mass of $M_{\star} \sim 10^{5-6} M_{\odot}$ (Farrell et al. 2009; Soria et al. 2010; Wiersema et al. 2010; Farrell et al. 2012; Soria et al. 2012; Farrell et al. 2014). The host cluster's observed mass resembles those of the probable GCs in M81, but the age of the host cluster's light-dominating stars, about $20 \mathrm{Myr}$, is significantly younger than GCs, which are older than 10 Gyr (Forbes et al. 2015). Still, the host cluster might be more massive and older than deduced so far, if it is actually the remnant of a stripped dwarf galaxy (Farrell et al. 2012, 2014). As the latter authors note, such extra mass could also help retain the gas from which the younger stars formed.

Near 7 GHz HLX-1 can achieve a flaring luminosity of $L_{\mathrm{R}} \sim 3.4 \times 10^{36} \mathrm{erg} \mathrm{s}^{-1}$ during transits from its hard to soft X-ray states (Webb et al. 2012), and a steady luminosity of $L_{\mathrm{R}} \sim 1.6 \times 10^{36} \mathrm{erg} \mathrm{s}^{-1}$ while in its hard X-ray state (Cseh et al. 2015). If that steady emission is Doppler boosted by a factor of about five to ten, as Cseh et al. (2015) argue, its side-on luminosity is about $L_{\mathrm{R}} \sim 1.6-3.2 \times 10^{35} \mathrm{erg} \mathrm{s}^{-1}$. Given these various radio 
luminosities for HLX-1, Figure 4 makes it clear that no HLX-1 analog resides in any of the 206 probable GCs in M81. Wrobel et al. (2015) found the same result for 337 candidate GCs in NGC 1023 and concluded that (i) HLX-1 is accreting gas related to the formation and/or presence of the 20-Myr-old stars in its host cluster or (ii) the HLX-1 phenomenon is just so rare that no radio analog is expected in NGC 1023. The same two conclusions apply in the case of M81.

\subsection{X-ray Detected Clusters}

Sell et al. (2011) used extensive Chandra data to localize several hundred X-ray sources in M81, and A. Zezas (2015, private communication) is investigating their matches with probable GCs. In the interim, we used version 1.1 of the Chandra Source Catalog (Evans et al. 2010) to identify X-ray sources within the optical positional uncertainty of M81's probable GCs. We also required that an X-ray detection had enough significance to establish a luminosity in the $0.5-10 \mathrm{keV}$ band. This led to detections of Nantais et al. (2011) IDs 273 and 284 as sources CXO J095547.0+690551 and CXO J095549.7+690531 with luminosities of $L_{\mathrm{X}} \sim 9.2 \times 10^{37} \mathrm{erg} \mathrm{s}^{-1}$ and $L_{\mathrm{X}} \sim 4.6 \times 10^{38} \mathrm{erg} \mathrm{s}^{-1}$, respectively.

These detections are consistent with prior Chandra results (Swartz et al. 2003; Liu 2011). Importantly, Liu (2011) noted that the two X-ray sources, detected in 16 or 17 observations, were always in a hard X-ray state, a requisite for applying the empirical fundamental-plane relation among the X-ray luminosity, $L_{\mathrm{X}}$, the radio luminosity, $L_{\mathrm{R}}$, and the $\mathrm{BH}$ mass, $M_{\mathrm{BH}}$. As we wish to estimate masses, we employ the Miller-Jones et al. (2012) regression of the contracted sample of Plotkin et al. (2012). Inserting the above values for $L_{\mathrm{X}}$ and Figure 4's upper limits to $L_{\mathrm{R}}$ into that regression, we estimate $\mathrm{BH}$ masses at $95 \%$ confidence of $M_{\mathrm{BH}}<99,000 M_{\odot}$ for ID 273 and $M_{\mathrm{BH}}<15,000 M_{\odot}$ for ID 284. The clusters' stellar masses are known from Figure 4, and lead to estimates for the BH mass fractions of $M_{\mathrm{BH}} / M_{\star}<0.18$ for ID 273 and $M_{\mathrm{BH}} / M_{\star}<0.21$ for ID 284. We comment further on these estimates in $\S 3.3$. As cautioned in $\S 1$, we cannot rule out contamination from X-ray-emitting stellar-mass BHs. Indeed, that is the more likely explanation because few IMBHs are known, whereas many $\mathrm{X}$-ray binaries are associated with extragalactic GCs.

It is also noteworthy that no probable GC in M81 has an X-ray luminosity as high as that of HLX-1 in its hard state, $L_{\mathrm{X}} \sim 2 \times 10^{40} \mathrm{erg} \mathrm{s}^{-1}$ (Godet et al. 2012). This absence of an X-ray analog of HLX-1 is consistent with the absence of a radio analog of HLX-1 (§ 3.1). However, since our limits on the radio luminosity are over an order of magnitude lower than even a de-boosted version of HLX-1, we are also able to rule out the presence of less extreme sources, even in the case where such a system was heavily absorbed and viewed edge-on, 
such that the X-ray emission would not be particularly remarkable.

\subsection{Semi-Empirical Model}

We adopt the semi-empirical model of Maccarone \& Servillat $(2008,2010)$ to predict the mass of a putative IMBH that, if experiencing hard-X-ray-state accretion in a GC, is consistent with the upper limit on the radio luminosity. Following Strader et al. (2012a) we conservatively assume that the IMBH in a GC accretes at a fraction $f_{b}=0.03$ of the Bondi rate from a medium with a gas density $\rho=0.2 \mathrm{~cm}^{-3}$. Such values yield a prediction for the hard-state X-ray luminosity $L_{\mathrm{X}}$. Then, the empirical fundamental-plane regression for the Plotkin et al. (2012) contracted sample is employed to predict the associated radio luminosity $L_{\mathrm{R}}$. In this way, a detection of, or upper limit to, a radio luminosity maps to a detection of, or upper limit to, an IMBH mass.

Figure 5 shows the results of applying this conservative, semi-emipirical model to the 206 probable GCs in M81. The best radio luminosity constraint for an individual GC implies a $3 \sigma \mathrm{IMBH}$ mass of $M_{\mathrm{BH}}<100,000 M_{\odot}$. To reach a lower mass regime, the AIPS task stack was used to form a weighted-mean image stack (e.g., Lindroos et al. 2015) of the cutouts of all 206 probable GCs. That stack, presented in Figure 6, measures the the mean contribution to the total radio emission from the probable GCs. For the all-cluster stack, the $3 \sigma$ radio-luminosity upper limit corresponds to an IMBH mass of $\overline{M_{\mathrm{BH}}(\text { all })}<42,000 M_{\odot}$.

However, the GCs' stellar masses, $M_{\star}$, range from about 10 thousand to 8 million solar masses, suggesting that it is physically more relevant to focus only on the more massive GCs. Like Wrobel et al. (2015), we thus focus on the GCs whose putative IMBHs have masses that are less than half the combined mass of their stars. The 49 GCs meeting that criterion are listed in Table 2 and plotted with dark symbols in Figure 5. The column contents of Table 2 are: column 1, the ID from Nantais et al. (2011); column 2, the stellar mass based on the V-band (F606W) magnitudes from Nantais et al. (2011) and that band's mass-to-light ratio for GCs from Harris et al. (2010); column 3, the IMBH mass based on the semi-empirical model; and column 4, the ratio of the IMBH mass in column 3 to the stellar mass in column 2. Each of the 49 massive GCs in Table 2 has a stellar mass $M_{\star} \gtrsim 200,000 M_{\odot}$. Fully 13 of these massive GCs have upper limits to their BH mass fractions, $M_{\mathrm{BH}} / M_{\star}$, in the range $0.03-0.15$. Such upper limits are thus below the remarkable value of 0.15 reported for M60-UCD1 (Seth et al. 2014).

The attributes of ID 273 in Table 2 deserve special mention: applying the semi-empirical model leads to estimates of a $\mathrm{BH}$ mass of $M_{\mathrm{BH}}<100,000 M_{\odot}$ and a $\mathrm{BH}$ mass fraction of 
$M_{\mathrm{BH}} / M_{\star}<0.19$. For comparison, applying the empirical fundamental plane, as in $\S 3.2$, led to estimates of $M_{\mathrm{BH}}<99,000 M_{\odot}$ and $M_{\mathrm{BH}} / M_{\star}<0.18$.

We also used the AIPS task stack to form a weighted-mean image stack of the cutouts of these 49 massive GCs. Figure 7 shows that stack, a measure of the the mean contribution to the total radio emission from the 49 massive GCs. The stack's $3 \sigma$ radio-luminosity upper limit corresponds to an IMBH mass of $\overline{M_{\mathrm{BH}} \text { (massive) }}<51,000 M_{\odot}$ for M81, a stronger constraint than the upper limit of 230,000 $M_{\odot}$ for NGC 1023 (Wrobel et al. 2015). The mean stellar mass of the massive GCs in M81 is $\overline{M_{\star} \text { (massive) }}=655,000 M_{\odot}$. Taking the ratio of these values leads to a $\mathrm{BH}$ mass fraction $\overline{M_{\mathrm{BH}} \text { (massive) }} / \overline{M_{\star} \text { (massive) }}<0.08$ for M81, improving over the equivalent fraction of less than 0.16 for NGC 1023 (Wrobel et al. 2015). The BH mass fraction of less than 0.08 for the massive GCs in M81 is well below the BH mass fraction of 0.15 for M60-UCD1 (Seth et al. 2014). Still, it should be kept in mind that the interpretation of the $\mathrm{BH}$ mass fraction for M81 is affected by unknowns like the distribution function of IMBH masses and the fraction of massive GCs occupied by an IMBH.

Within the context of this semi-empirical model, the VLA constraints on individual GCs and stacks of GCs are beginning to probe the domain of IMBHs in extragalactic GCs. These constraints for M81 can be improved with longer VLA exposures or similar exposures with the next-generation VLA (ngVLA; Carilli et al. 2015). For perspective, assuming a sufficient dynamic range given M81's LLAGN, a one-hour exposure with the ngVLA at a frequency of $10 \mathrm{GHz}$ could reach an rms noise of $0.45 \mu \mathrm{Jy}_{\text {beam }^{-1}}$ for an individual GC. Such an ngVLA value would be very close to the rms noise achieved in Figure 6 only after stacking the VLA images of all 206 probable GCs in M81.

\section{Summary and Conclusions}

We used a four-pointing VLA mosaic at $5.5 \mathrm{GHz}$ to search for the radiative signatures of IMBH accretion from 206 probable GCs in M81, a spiral galaxy at a distance of 3.63 Mpc. None of the individual GCs were detected. Similarly, only upper limits were obtained from weighted-mean image stacks of all 206 GCs and of the 49 massive GCs with $M_{\star} \gtrsim$ $200,000 M_{\odot}$. We examined the implications of these data for IMBHs, if any exist in these GCs. Our principal findings are as follows:

1. The 206 GCs in M81 lack radio analogs of HLX-1, a strong IMBH candidate in a star cluster in the early-type galaxy ESO 243-49. This suggests that HLX-1 is accreting gas related to the 20-Myr-old stars in its host cluster or that the HLX-1 phenomenon is so 
rare that no radio analog is expected in M81.

2. Two GCs exhibit hard-state X-ray emission. From the empirical fundamental-plane relation, their X-ray and radio luminosities suggest individual IMBH masses, $M_{\mathrm{BH}}$, of less than 99,000 $M_{\odot}$ and 15,000 $M_{\odot}$, and associated $\mathrm{BH}$ mass fractions, $M_{\mathrm{BH}} / M_{\star}$, of less than 0.18 and 0.21 . With only upper limits to the radio luminosities, we cannot rule out the likely scenario of contamination from X-ray-emitting stellar-mass BHs in these GCs.

3. A semi-empirical model developed for Milky Way GCs converts the upper limits on radio luminosities to upper limits on IMBH masses. Applying this model to M81, over a dozen individual GCs appear to have upper limits on the BH mass fractions, $M_{\mathrm{BH}} / M_{\star}$, that are below the noteworthy value of 0.15 reported for M60-UCD1. Also, the M81 stacks correspond to IMBH masses of $\overline{M_{\mathrm{BH}}(\text { all })}<42,000 M_{\odot}$ for all the GCs and to $\overline{M_{\mathrm{BH}} \text { (massive) }}<51,000 M_{\odot}$ for the massive GCs. This model is making inroads into the difficult-to-observe regime of IMBHs in extragalactic GCs.

We thank the referee for a helpful and timely report, and Dr. E. Greisen for providing the new AIPS task stack. JCAMJ is the recipient of an Australian Research Council Future Fellowship (FT140101082). MJM acknowledges an Ernest Rutherford STFC fellowship (grant number RG77623). This research has made use of data obtained from the Chandra Source Catalog, provided by the Chandra X-ray Center (CXC) as part of the Chandra Data Archive. This research has made use of the NASA/IPAC Extragalactic Database (NED) which is operated by the Jet Propulsion Laboratory, California Institute of Technology, under contract with the National Aeronautics and Space Administration. Any opinions, findings, and conclusions or recommendations expressed in this material are those of the authors and do not necessarily reflect the views of the National Science Foundation.

Facilities: CXO, VLA.

\section{REFERENCES}

Abbott, B. P., Abbott, R., Abbott, T. D., et al. 2016a, PhRvL, 116, 061102

Abbott, B. P., Abbott, R., Abbott, T. D., et al. 2016b, ApJ, 818, L22

Carilli, C. L., McKinnon, M., Ott, J., et al. 2015, Next Generation Very Large Array Memo No. 5, Science Working Groups Project Overview, arXiv:1510.06438 
Chandar, R., Ford, H. C., \& Tsvetanov, Z. 2001, AJ, 122, 1330

Chandar, R., Whitmore, B., \& Lee, M. G. 2004, ApJ, 611, 220

Corral-Santana, J. M., Casares, J., Munoz-Barias, T., et al. 2016, A\&A, 587, A61

Cseh, D., Webb, N. A., Godet, O., et al. 2015, MNRAS, 446, 3268

Do, T., Wright, S., Barth, A. J., et al. 2014, AJ, 147, 93

Evans, I. N., Primini, F. A., Glotfelty, K. J., et al. 2010, ApJS, 189, 37

Falcke, H., Kording, E., \& Markoff, S. 2004, A\&A, 414, 895

Farrell, S. A., Webb, N. A., Barret, D., Godet, O., \& Rodrigues, J. M. 2009, Nature, 460, 73

Farrell, S. A., Servillat, M., Pforr, J., et al. 2012, ApJ, 747, L13

Farrell, S. A., Servillat, M., Gladstone, J. C., et al. 2013, MNRAS, 437, 1208

Fender, R., \& Belloni, T. 2012, Science, 337, 540

Forbes, D. A., Pastorello, N., Romanowsky, A. J., et al. 2015, MNRAS, 452, 1045

Freedman, W. L., Hughes, S. M., Madore, B. F., et al. 1994, ApJ, 427, 628

Gebhardt, K., Rich, R. M., \& Ho, L. C. 2005, ApJ, 634, 1093

Giersz, M., Leigh, N., Hypki, A. Ltzgendorf, N., \& Askar, A. 2015, MNRAS, 454, 315

Godet, O., Plazolles, B., Kawaguchi, T., et al. 2012, ApJ, 752, 34

Graham, A. W., Ciambur, B. C., \& Soria, R. 2016, ApJ, 818, 172

Greene, J. E. 2012, Nat. Comm., 3, 1304

Griesen, E. W. 2003, in Information Handling in Astronomy, ed. A. Heck (Dordrecht: Kluwer), 109

Gurkan, M. A., Freitag, M., \& Rasio, F. A. 2004, ApJ, 604, 632

Harris, W. E., Spitler, L. R., Forbes, D. A., \& Bailin, J. 2010, MNRAS, 401, 1965

Jarrett, T. H., Chester, T., Cutri, R., Schneider, S. E., \& Huchra, J. P. 2003, AJ, 125, 525

Jiang, Y. F., Greene, J. E., Ho, L. C., et al. 2011, ApJ, 742, 68 
Konstantinidis, S., Amaro-Seoane, P., \& Kokkotas, K. D. 2013, A\&A, 557, a135

Kormendy, J., \& Ho, L. C. 2013, ARA\&A, 51, 511

Lindroos, L., Knudsen, K. K., Vlemmings, W., Conway, J., \& Marti-Vidal, I. 2015, MNRAS, 446, 3502

Liu, J. 2011, ApJS, 192, 10

Maccarone, T. J. 2004, MNRAS, 351, 1049

Maccarone, T. J., \& Servillat, M. 2008, MNRAS, 389, 379

Maccarone, T. J., \& Servillat, M. 2010, MNRAS, 408, 2511

Mayya, Y. D., Rosa-Gonzalez, D., Santiago-Cortes, M., et al., 2013, MNRAS, 436, 2763

McMullin, J. P., Waters, B., Schiebel, D., Young, W., \& Golap, K. 2007, in ASP Conf. Ser. 376, Astronomical Data Analysis Software and Systems, IVI, ed. R. A. Shaw, F. Hill, \& D. J. Bell (San Francisco, CA: ASP), 127

Merloni, A., Heinz, S., \& DiMatteo, T. 2003, MNRAS, 345, 1057

Middleton, M. J., Miller-Jones, J. C. A., Markoff, S., et al. 2013, Nature, 493, 187

Middleton, M. J., Miller-Jones, J. C. A., \& Fender, R. P. 2014, MNRAS, 439, 1740

Miller, M. C., \& Hamilton, D. P. 2002, MNRAS, 330, 232

Miller-Jones, J. C. A., Wrobel, J. M., Sivakoff, G. R., et al. 2012, ApJ, 755, L1

Nantais, J. B., Huchra, J. P., McLeod, B., Strader, J., \& Brodie, J. P. 2010, AJ, 139, 1413

Nantais, J. B., Huchra, J. P., Zezas, A., Gazeas, K., \& Strader, J. 2011, AJ, 142, 183

Natarajan, P. 2014, General Relativity and Gravitation, 46, 1072

Perelmuter, J. M., \& Racine, R. 1995, AJ, 109, 1055

Perley, R. A., Chandler, C. C., Butler, B. J., \& Wrobel, J. M. 2011, ApJ, 739, L1

Plotkin, R. M., Markoff, S., Kelly, B. C., Koerding, E., \& Anderson, S. F. 2012, MNRAS, 419, 267

Portegies Zwart, S. F., Baumgardt, H., Hut, P., Makino, J., \& McMillian, S. L. W. 2004, Nature, 428, 724 
Santiago-Cortes, M., Mayya, Y. D., \& Rosa-Gonzalez, D. 2010, MNRAS, 405,1293

Sell, P. H., Pooley, D., Zezas, A., et al. 2011, ApJ, 735, 26

Seth, A. C., van den Bosch, R., Mieske, S., et al. 2014, Nature, 513, 398

Soria, R., Hau, G. K. T., Graham, A. W., et al. 2010, MNRAS, 405, 870

Soria, R., Hakala, P. J., Hau, G. K. T., et al. 2010, MNRAS, 420, 3599

Strader, J., Chomiuk, L., Maccarone, T. J., et al. 2012a, ApJ, 750, L27

Strader, J., Chomiuk, L., Maccarone, T. J., Miller-Jones, J. C. A., \& Seth, A. C. 2012b, Nature, 490, 71

Jun, M. Y., Jin, Y. L., Gu, W. M., et al. 2013, ApJ, 776, 118

Swartz, D. A., Ghosh, K. K., McCollough, M. L., et al. 2003, ApJS, 144, 213

Tetarenko, B. E., Sivakoff, G. R., Heinke, C. O., \& Gladstone, J. C. 2016, ApJS, 222, 15

Volonteri, M. 2012, Science, 337, 544

Wall, J. V., \& Jenkins, C. R. 2003, Practical Statistics for Astronomers, Cambridge: CUP, Table A2.2

Webb, N., Cseh, D., Lenc, E., et al. 2012, Science, 337, 554

Wiersema, K., Farrell, S. A., Webb, N. A., et al. 2010, ApJ, 721, L102

Wrobel, J. M., Nyland, K. E., \& Miller-Jones, J. C. A. 2015, AJ, 150, 120

Xiao, T., Barth, A. J., Greene, J. E., et al. 2011, ApJ, 739, 28 


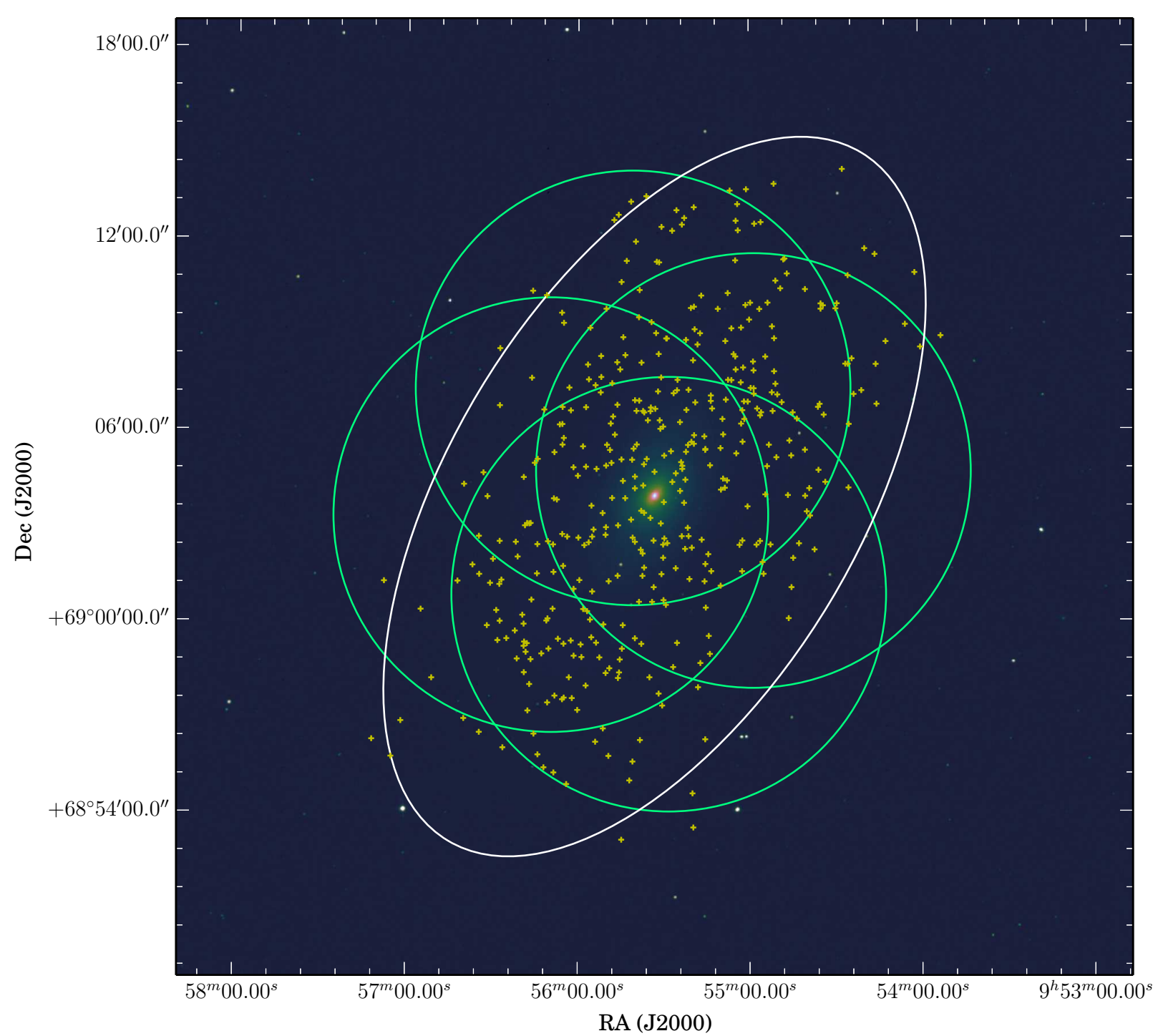

Fig. 1. - Four overlapping green circles indicate the VLA mosaic region for M81 at 5.5 GHz. Each circle is located at one of the VLA pointing centers (Table 1) and has a diameter of $13 ! 6(14.4 \mathrm{kpc})$, where the response of the VLA primary beam falls to the $10 \%$ level. The colored shading shows the surface brightness of the inner galaxy in the $K_{s}$ band, while the white ellipse conveys the extent of the galaxy in that band as deduced by Jarrett et al. (2003). The yellow plus signs mark the locations of the 419 candidate GCs from Nantais et al. (2011). 


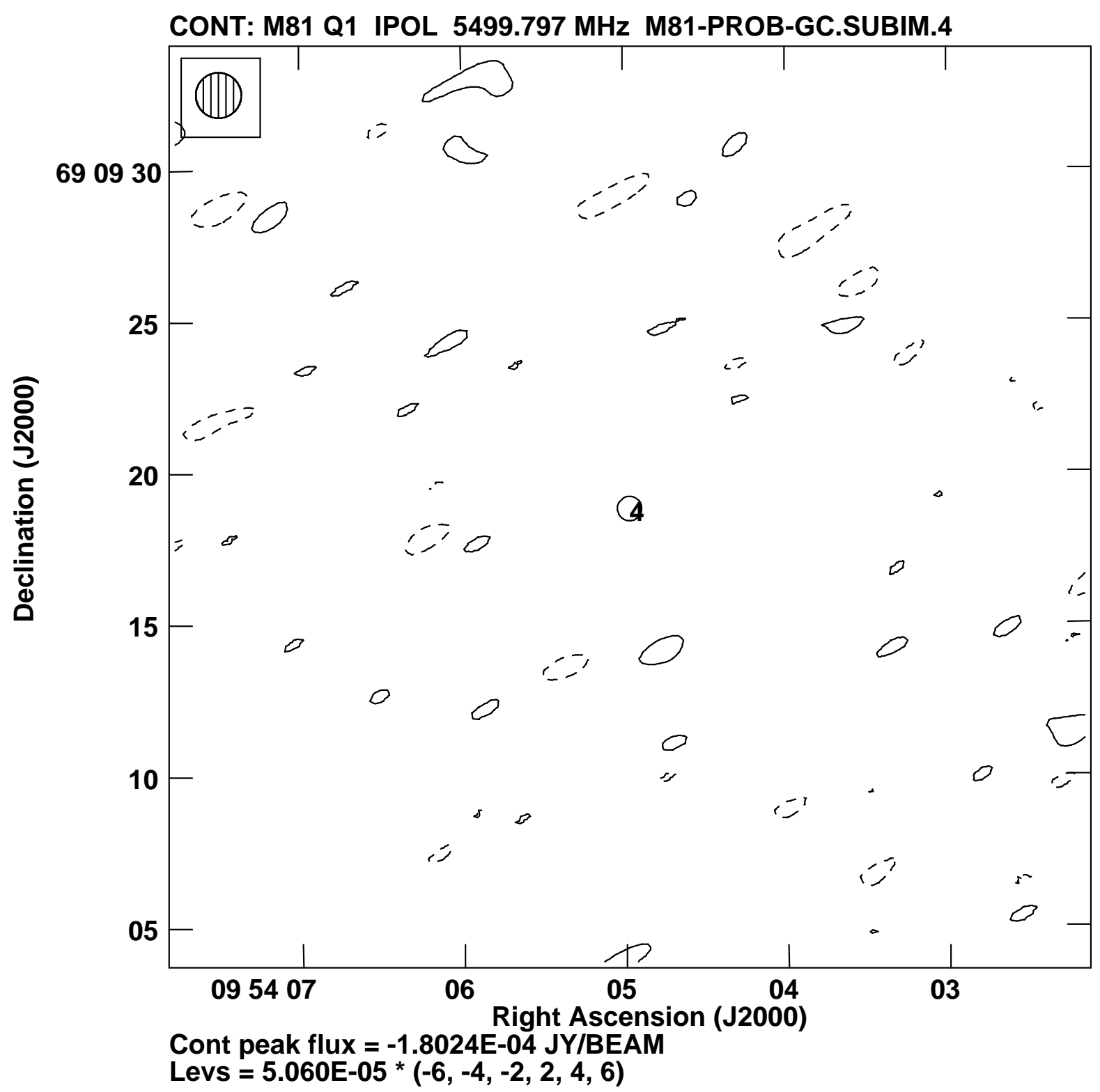

Fig. 2.- VLA cutout of the Stokes $I$ emission at $5.5 \mathrm{GHz}$ centered on the optical position of a probable GC in M81. The cutout spans $30^{\prime \prime}(1.6 \mathrm{kpc})$ per coordinate. The diameter of the hatched circle in the north-east corner, 1".5 (26.4 pc), is the FWHM of the VLA synthesized beam. Contours are at $-6,-4,-2,2,4$, and 6 times the $1 \sigma$ rms noise shown in the legend in units of $\mathrm{Jy} \mathrm{beam}^{-1}$. Linearly-spaced contours are chosen to convey noise levels at a glance, with dashed lines showing negative contours and solid lines showing positive ones. The central circle of diameter 0 ".8 (32 pc) shows the cluster's optical positional uncertainty at $90 \%$ confidence and is labelled with its ID from Nantais et al. (2011). The VLA photometry seeks evidence for the accretion signature of a point-like IMBH in the cluster's center. The cluster is not detected above the $3 \sigma$ level. Figures $2.1-2.206$ are available, ordered by increasing ID, in the online version of the Journal. Some cutouts show additional candidate GCs offset from the central probable GC. 


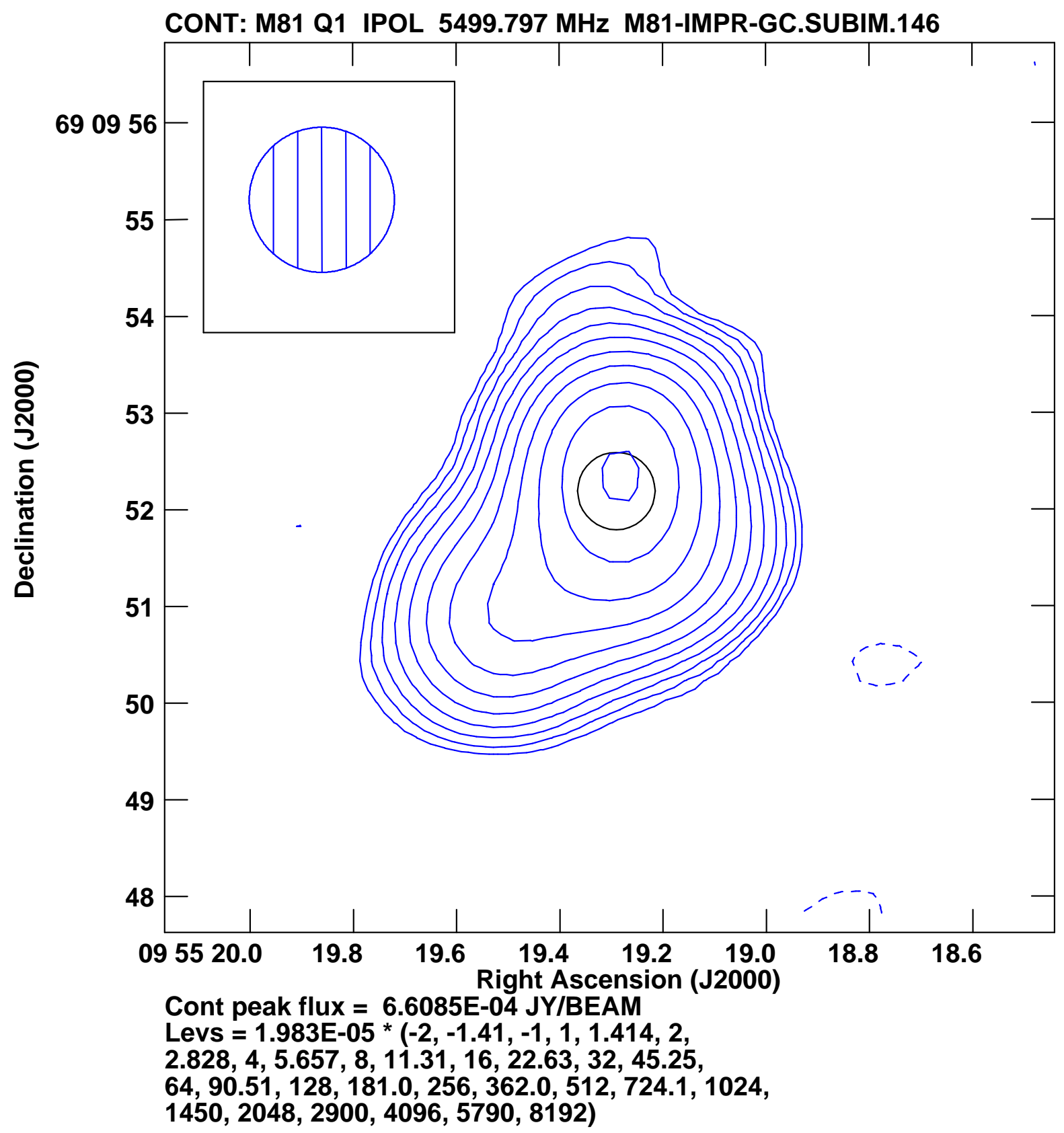

Fig. 3.- Zoom in of the VLA cutout of the Stokes $I$ emission at $5.5 \mathrm{GHz}$ centered on the optical position of ID 146, an improbable GC in M81 (Nantais et al. 2011). The diameter of the hatched circle in the north-east corner, 1".5, is the FWHM of the VLA synthesized beam. Blue contour levels are at intervals of $\sqrt{2}$ times the bottom contour level, which is $3 \%$ of the image peak. Negative contours are dashed and positive ones are solid. The black central circle of diameter 0 " 8 shows the optical positional uncertainty at $90 \%$ confidence. A linear scale is not given because ID 146 might be a background galaxy. 
206 Probable Globular Clusters in M81

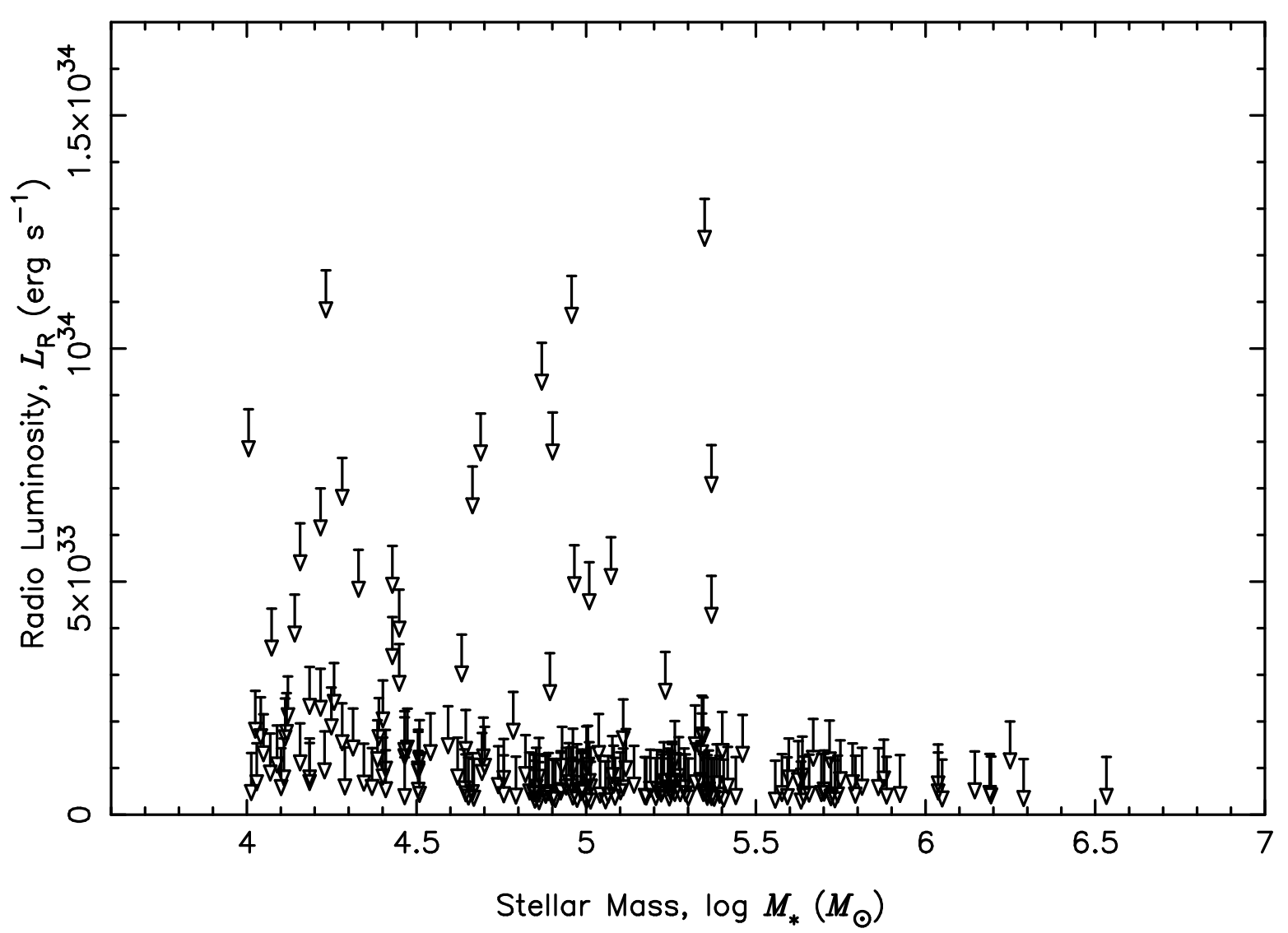

Fig. 4.- Upper limits to the radio luminosities at $5.5 \mathrm{GHz}, L_{\mathrm{R}}(3 \sigma)$, as a function of the stellar masses, $M_{\star}$, of 206 individual probable GCs in M81. 
206 Probable Globular Clusters in M81

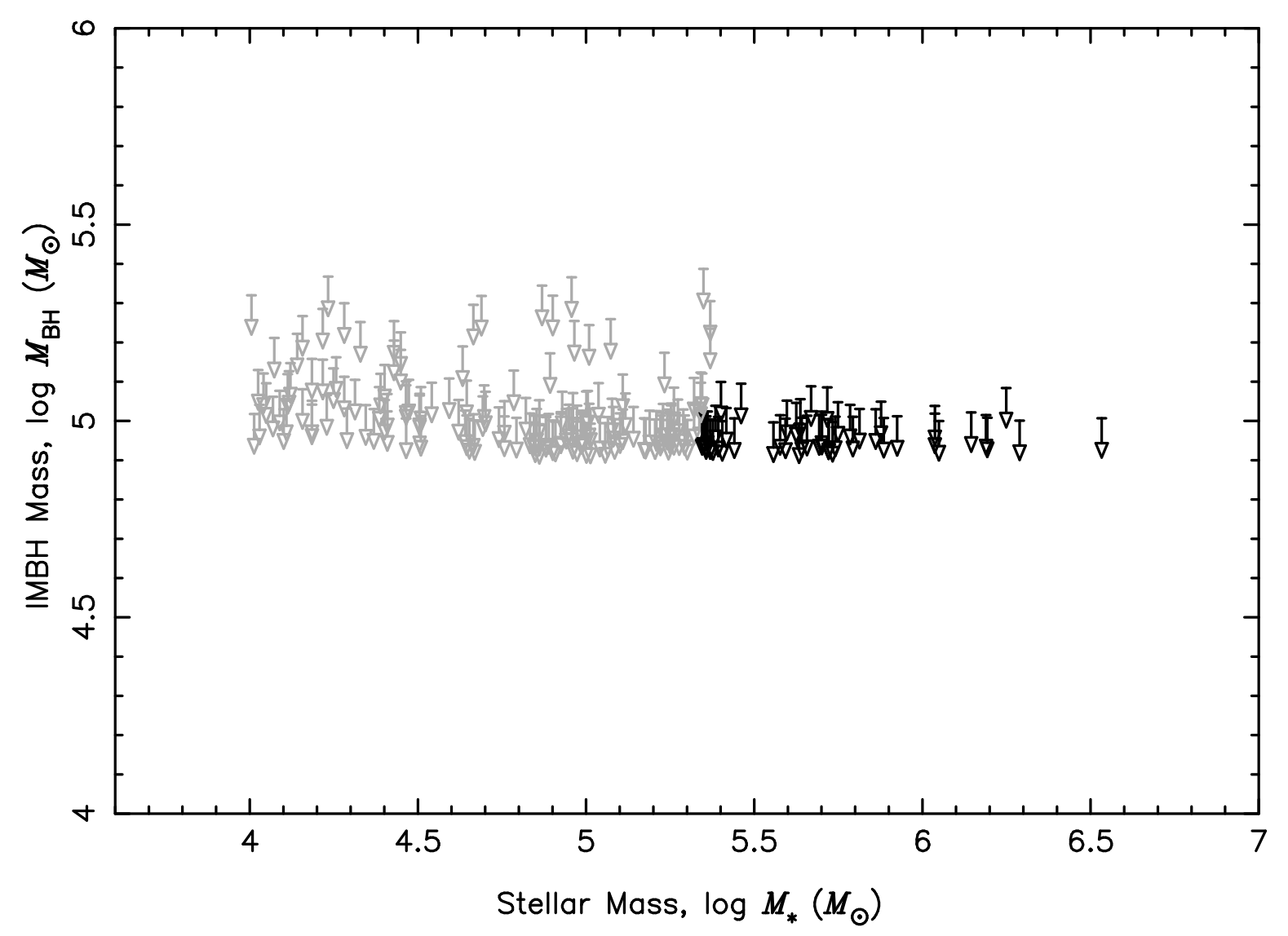

Fig. 5.- Upper limits to the mass of the IMBH, $M_{\mathrm{BH}}(3 \sigma)$, as a function of the stellar masses, $M_{\star}$, of 206 individual probable GCs in M81. A semi-empirical model uses the radio luminosity to infer the mass of the IMBH, assuming one exists. The dark symbols highlight the 49 massive GCs with $\mathrm{BH}$ mass fractions $M_{\mathrm{BH}} / M_{\star}<0.5$. 


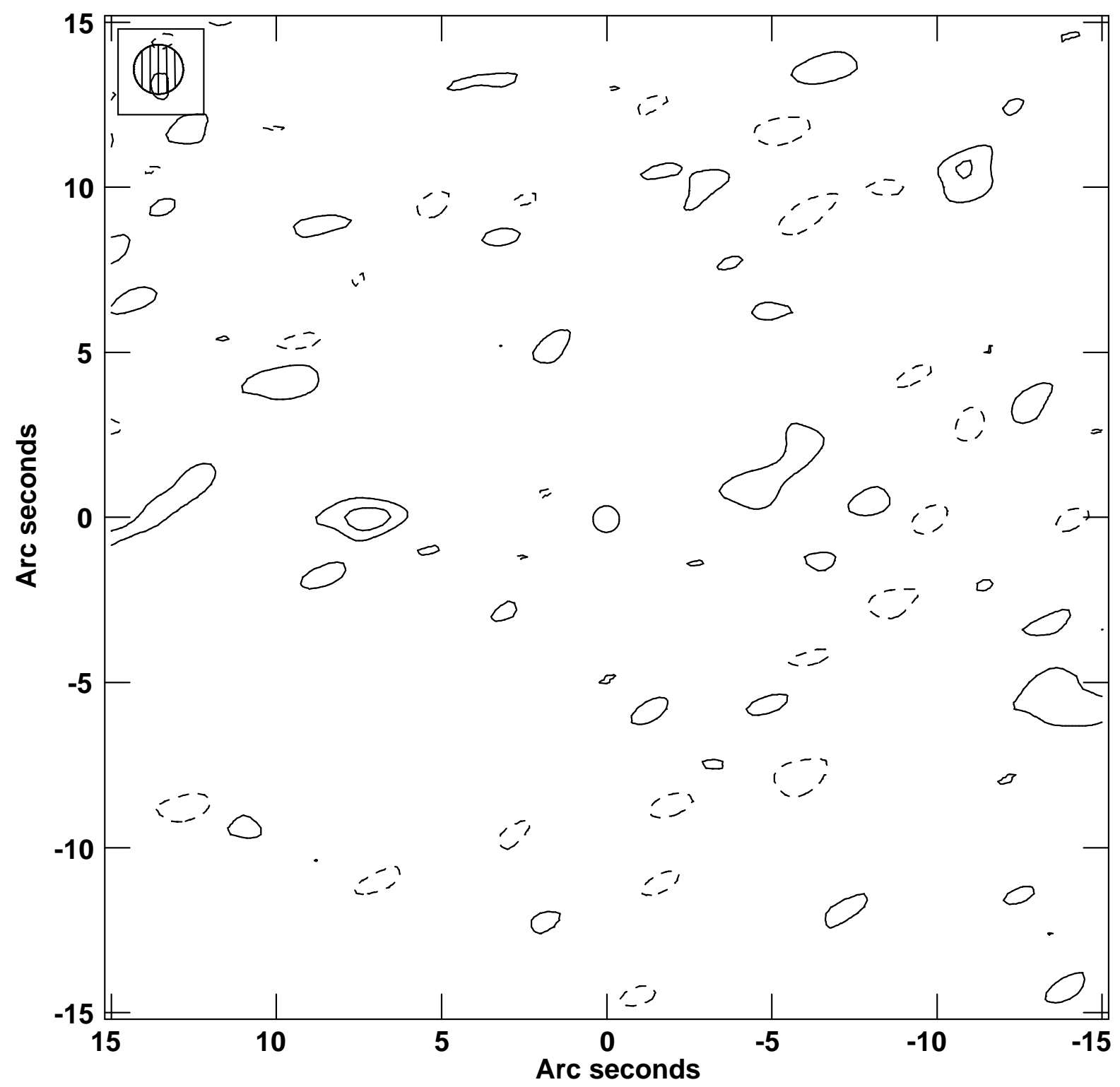

Fig. 6.- Weighted-mean stack of the VLA images of the 206 probable GCs. The stacked image has an rms noise of $0.43 \mu \mathrm{Jy}$ beam $^{-1}(1 \sigma)$. The hatched circle, central circle and contouring scheme are the same as for Figure 2. No emission is detected above $3 \sigma=$ $1.29 \mu \mathrm{Jy}_{\text {beam }^{-1}}$. 


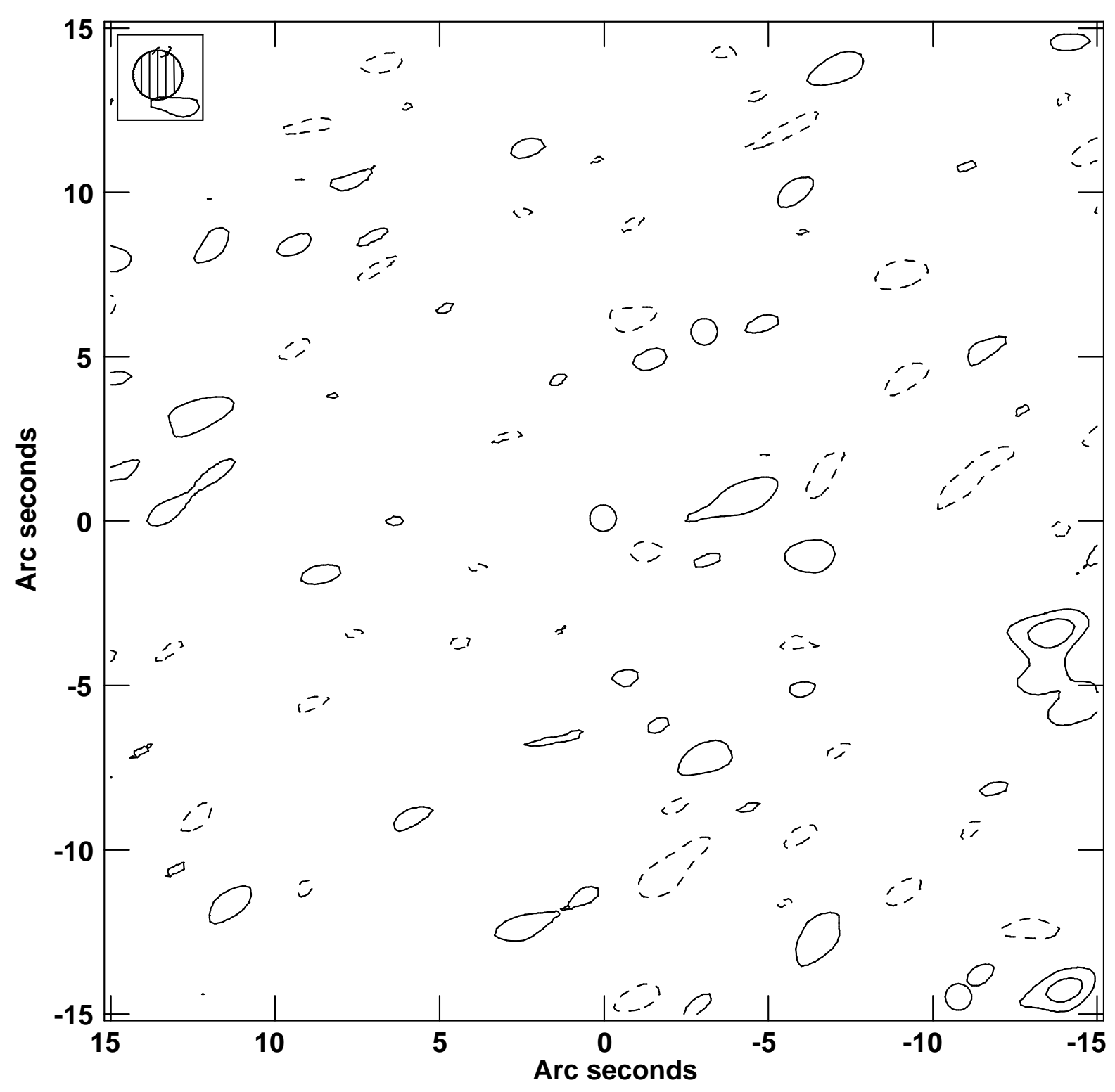

Fig. 7.- Weighted-mean stack of the VLA images of the 49 massive GCs. The stacked image has an rms noise of $0.74 \mu \mathrm{Jy}_{\text {beam }^{-1}}(1 \sigma)$. The hatched circle, central circle and contouring scheme are the same as for Figure 2. No emission is detected above $3 \sigma=2.22 \mu \mathrm{Jy} \mathrm{beam}^{-1}$. 
Table 1. VLA Pointing Centers and Observation Dates

\begin{tabular}{cccc}
\hline \hline $\begin{array}{c}\text { R.A. } \\
(J 2000)\end{array}$ & $\begin{array}{c}\text { Decl. } \\
(\mathrm{J} 2000)\end{array}$ & UT Date & MJD \\
\hline 095540.71 & 6907 19.45 & 2014 Jan 5 & $56662.15 \pm 0.02$ \\
095458.45 & 690443.90 & 2014 Jan 6 & $56663.11 \pm 0.02$ \\
095609.55 & 690320.83 & 2014 Jan 5 & $56662.12 \pm 0.02$ \\
095528.20 & 690051.34 & 2014 Jan 6 & $56663.16 \pm 0.02$ \\
\hline
\end{tabular}

Note. - Units of right ascension are hours, minutes, and seconds, and units of declination are degrees, arcminutes, and arcseconds. 
Table 2. Massive Globular Clusters in M81

\begin{tabular}{rccc}
\hline \hline $\mathrm{ID}$ & $M_{\star}$ & $M_{\mathrm{BH}}$ & $M_{\mathrm{BH}} / M_{\star}$ \\
& $\left(M_{\odot}\right)$ & $\left(M_{\odot}\right)$ & \\
\hline 28 & $0.52 \mathrm{E}+06$ & $<0.12 \mathrm{E}+06$ & $<0.23$ \\
31 & $0.43 \mathrm{E}+06$ & $<0.11 \mathrm{E}+06$ & $<0.26$ \\
71 & $0.26 \mathrm{E}+06$ & $<0.11 \mathrm{E}+06$ & $<0.41$ \\
90 & $0.11 \mathrm{E}+07$ & $<0.10 \mathrm{E}+06$ & $<0.10$ \\
100 & $0.28 \mathrm{E}+06$ & $<0.10 \mathrm{E}+06$ & $<0.37$ \\
115 & $0.39 \mathrm{E}+06$ & $<0.10 \mathrm{E}+06$ & $<0.26$ \\
116 & $0.44 \mathrm{E}+06$ & $<0.10 \mathrm{E}+06$ & $<0.23$ \\
118 & $0.19 \mathrm{E}+07$ & $<0.10 \mathrm{E}+06$ & $<0.05$ \\
136 & $0.23 \mathrm{E}+06$ & $<0.10 \mathrm{E}+06$ & $<0.44$ \\
145 & $0.25 \mathrm{E}+06$ & $<0.10 \mathrm{E}+06$ & $<0.39$ \\
158 & $0.53 \mathrm{E}+06$ & $<0.10 \mathrm{E}+06$ & $<0.19$ \\
160 & $0.34 \mathrm{E}+07$ & $<0.10 \mathrm{E}+06$ & $<0.03$ \\
162 & $0.11 \mathrm{E}+07$ & $<0.10 \mathrm{E}+06$ & $<0.09$ \\
175 & $0.45 \mathrm{E}+06$ & $<0.10 \mathrm{E}+06$ & $<0.23$ \\
190 & $0.53 \mathrm{E}+06$ & $<0.10 \mathrm{E}+06$ & $<0.19$ \\
199 & $0.11 \mathrm{E}+07$ & $<0.11 \mathrm{E}+06$ & $<0.10$ \\
201 & $0.23 \mathrm{E}+06$ & $<0.10 \mathrm{E}+06$ & $<0.43$ \\
209 & $0.62 \mathrm{E}+06$ & $<0.10 \mathrm{E}+06$ & $<0.16$ \\
218 & $0.47 \mathrm{E}+06$ & $<0.12 \mathrm{E}+06$ & $<0.26$ \\
226 & $0.22 \mathrm{E}+06$ & $<0.10 \mathrm{E}+06$ & $<0.47$ \\
227 & $0.49 \mathrm{E}+06$ & $<0.10 \mathrm{E}+06$ & $<0.21$ \\
228 & $0.84 \mathrm{E}+06$ & $<0.10 \mathrm{E}+06$ & $<0.12$ \\
232 & $0.65 \mathrm{E}+06$ & $<0.11 \mathrm{E}+06$ & $<0.17$ \\
236 & $0.22 \mathrm{E}+06$ & $<0.10 \mathrm{E}+06$ & $<0.47$ \\
239 & $0.25 \mathrm{E}+06$ & $<0.10 \mathrm{E}+06$ & $<0.41$ \\
246 & $0.15 \mathrm{E}+07$ & $<0.10 \mathrm{E}+06$ & $<0.07$ \\
247 & $0.24 \mathrm{E}+06$ & $<0.10 \mathrm{E}+06$ & $<0.42$ \\
256 & $0.36 \mathrm{E}+06$ & $<0.99 \mathrm{E}+05$ & $<0.28$ \\
269 & $0.16 \mathrm{E}+07$ & $<0.10 \mathrm{E}+06$ & $<0.07$ \\
273 & $0.55 \mathrm{E}+06$ & $<0.10 \mathrm{E}+06$ & $<0.19$
\end{tabular}


Table 2-Continued

\begin{tabular}{cccc}
\hline \hline ID & $M_{\star}$ & $M_{\mathrm{BH}}$ & $M_{\mathrm{BH}} / M_{\star}$ \\
& $\left(M_{\odot}\right)$ & $\left(M_{\odot}\right)$ & \\
\hline 275 & $0.50 \mathrm{E}+06$ & $<0.10 \mathrm{E}+06$ & $<0.21$ \\
277 & $0.43 \mathrm{E}+06$ & $<0.99 \mathrm{E}+05$ & $<0.23$ \\
282 & $0.50 \mathrm{E}+06$ & $<0.11 \mathrm{E}+06$ & $<0.21$ \\
288 & $0.29 \mathrm{E}+06$ & $<0.12 \mathrm{E}+06$ & $<0.43$ \\
292 & $0.54 \mathrm{E}+06$ & $<0.99 \mathrm{E}+05$ & $<0.18$ \\
293 & $0.61 \mathrm{E}+06$ & $<0.11 \mathrm{E}+06$ & $<0.18$ \\
294 & $0.75 \mathrm{E}+06$ & $<0.11 \mathrm{E}+06$ & $<0.15$ \\
295 & $0.23 \mathrm{E}+06$ & $<0.11 \mathrm{E}+06$ & $<0.46$ \\
301 & $0.77 \mathrm{E}+06$ & $<0.10 \mathrm{E}+06$ & $<0.13$ \\
302 & $0.73 \mathrm{E}+06$ & $<0.11 \mathrm{E}+06$ & $<0.15$ \\
304 & $0.23 \mathrm{E}+06$ & $<0.10 \mathrm{E}+06$ & $<0.45$ \\
307 & $0.40 \mathrm{E}+06$ & $<0.11 \mathrm{E}+06$ & $<0.28$ \\
315 & $0.38 \mathrm{E}+06$ & $<0.10 \mathrm{E}+06$ & $<0.27$ \\
330 & $0.24 \mathrm{E}+06$ & $<0.11 \mathrm{E}+06$ & $<0.45$ \\
340 & $0.42 \mathrm{E}+06$ & $<0.11 \mathrm{E}+06$ & $<0.26$ \\
351 & $0.14 \mathrm{E}+07$ & $<0.11 \mathrm{E}+06$ & $<0.08$ \\
388 & $0.56 \mathrm{E}+06$ & $<0.11 \mathrm{E}+06$ & $<0.20$ \\
398 & $0.18 \mathrm{E}+07$ & $<0.12 \mathrm{E}+06$ & $<0.07$ \\
410 & $0.25 \mathrm{E}+06$ & $<0.13 \mathrm{E}+06$ & $<0.50$ \\
\hline
\end{tabular}

